\title{
Auto-fluorescent PAMAM-based dendritic molecules and their potential application in pharmaceutical sciences
}

\author{
Alaa M. M. El-Betany,, b,c, * Elbadawy A. Kamoun, ${ }^{\text {d,e }}$ Craig James, ${ }^{\mathrm{b}}$ Abdulhakim Jangher, ${ }^{\mathrm{b}, \mathrm{f}}$ \\ Ghaith Aljayyoussi, ${ }^{\text {a,g }}$ Peter Griffiths, ${ }^{\text {b,h }}$ Neil B. McKeown ${ }^{\text {,i, }}$ and Mark Gumbleton ${ }^{\text {a }}$
}

a School of Pharmacy and Pharmaceutical Sciences, Cardiff University, Cardiff, CF10 3NB, United Kingdom.

${ }^{\mathrm{b}}$ School of Chemistry, Cardiff University, Cardiff, CF10 3AT, United Kingdom.

${ }^{c}$ Chemistry Department, Faculty of Science, Damietta University, New Damietta City, 34517, Egypt.

d Polymeric Materials Research Dep., Advanced Technology and New Materials Research Institute (ATNMRI),

City of Scientific Research and Technological Applications (SRTA-City), New Borg Al-Arab 21934,

Alexandria, Egypt.

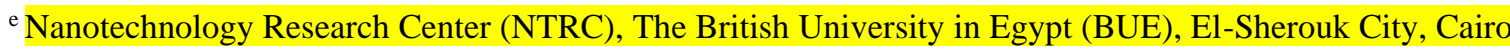
11837, Egypt.

${ }^{\mathrm{f}}$ Chemistry Department, Faculty of Science, Tripoli University, Tripoli City, 34517, Libya.

g Research Centre for Drugs and Diagnostics, Liverpool School of Tropical Medicine, Liverpool, L3 5QA, UK

${ }^{\mathrm{h}}$ Faculty of Engineering and Science, University of Greenwich, Kent, ME4 4TB, United Kingdom.

${ }^{i}$ EastChem, School of Chemistry, University of Edinburgh, Edinburgh EH9 3FJ, United Kingdom.

\section{Graphical Abstract}
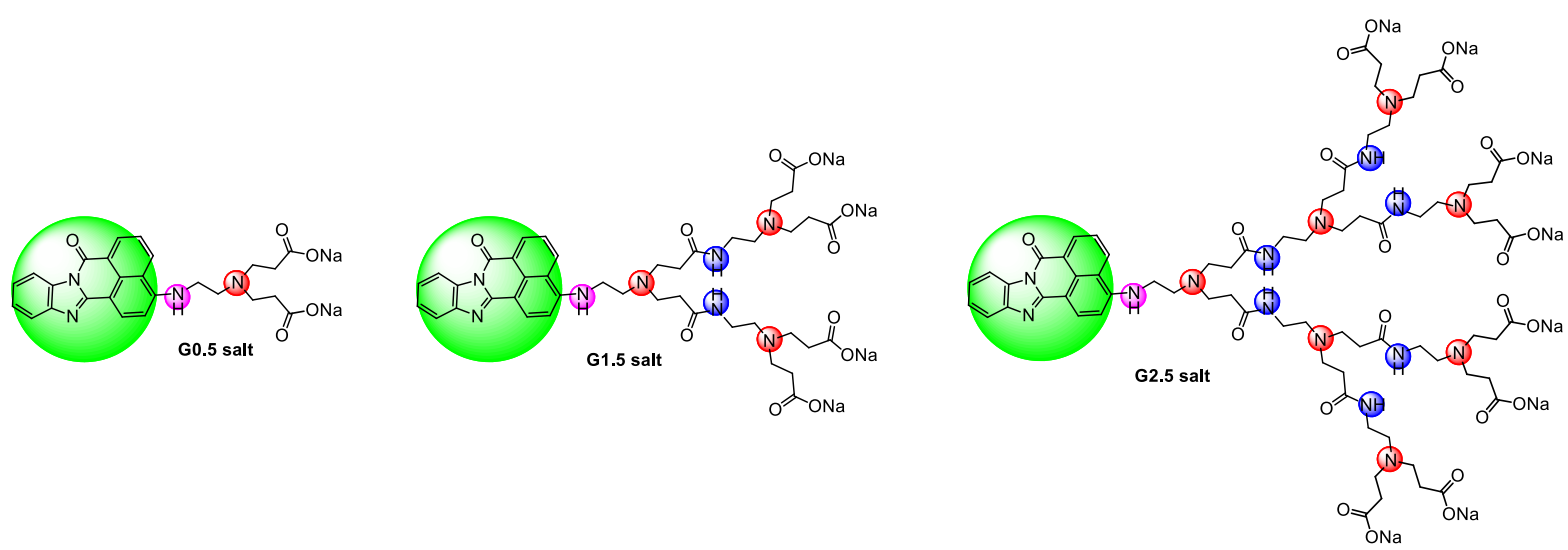


\begin{abstract}
The epithelial permeation of water-soluble fluorescent PAMAM dendrons based on 7Hbenz[de] benzimidazo [2,1-a] isoquinoline-7-one as a fluorescent core across epithelial cell models MDCK I and MDCK II has been quantified.

Hydrodynamic radii have been derived from self-diffusion coefficients obtained via pulsedgradient spin-echo Nuclear Magnetic Resonance (PGSE-NMR). Results indicate that these dendritic molecules are molecularly disperse, non-aggregating, and only slightly larger than their parent homologues. MDCK I permeability studies across epithelial barriers show that these dendritic molecules are biocompatible with the chosen epithelial in-vitro model and can permeate across MDCK cell monolayers. Permeability is demonstrated to be a property of dendritic size and cell barrier restrictiveness indicating that paracellular mechanisms play the predominant role in the transport of these molecules.
\end{abstract}

Keywords: PAMAM dendrons, water-soluble fluorescent dendrons, self-diffusion coefficient, hydrodynamic radii, permeability, imaging and visualisation. 


\section{Introduction}

There is continuing interest in water-soluble fluorescent dendrimers for biological applications for visualization and diagnostic imaging purposes.[1-6] Fluorescent labels are required to have high sensitivity, appropriate excitation and emission maxima, and specific functionality. It is these requirements that necessitate the development of new fluorescent labels with high detection limits and appropriate fluorescent behaviour.[7] Self-quenching is one of the key challenges in aqueous media due to the aggregation of the often hydrophobic fluorophore. Hence, it has been found beneficial to use bulky water-soluble dendritic structures to hinder self-association.[6] Fluorescent units can be incorporated within a dendrimer as either peripheral groups, branch points or as the core. It is clear that the location of the fluorophore influences its properties but also has a major role in directing the method and complexity of the synthesis of the dendrimer [6]. The loading of fluorophores onto the periphery of dendrimers can present many technical complexities including difficulties in the stoichiometric control of the synthesis.[8,9] Intramolecular fluorescent quenching issues are also more likely to occur when the fluorescent label is attached to the dendrimer's periphery, leading to the reduced overall fluorescent activity of the dendrimer.[6, 8, 9] These disadvantages may be avoided by incorporating the fluorophore as the core of a dendrimer or at the focal point of a dendritic molecule (dendron).

Due to their strong fluorescence and high photostability, derivatives of 1,8-naphthalimide are widely used as fluorescent markers in biology,[10] light-emitting diodes,[11-13] photoinduced electron transfer sensors,[14-18] fluorescent switches,[19-21] and photochemotherapeutic inhibitors.[22-26] A number of 1,8-naphthalimide derivatives were screened for fluorescence and $7 H$-benz[de]benzimidazo[2,1-a]isoquinoline-7-one (BBIQ) selected for use as the fluorophore due to its strong fluorescence (as assessed by relative fluorescence quantum yields).[7, [27]-43]

Since their development in the mid-1980s, PAMAM dendrimers have shown great potential in being utilised as drug delivery vehicles due to their biocompatibility, low polydispersity indices and high hydrophilicity. PAMAM dendrimers can additionally mimic globular proteins,[28] are readily transported across the epithelial barrier of the gut,[29-31] inhibit protein-protein binding,[32] and can be tailored to carry multiple cargo due to their high multi-valency. While, in general, PAMAM dendrimers are of biomedical interest, it is the anionic carboxylateterminated PAMAM dendrimers which are considered to be of greater potential as drug delivery vehicles given their high biocompatibility in contrast to the high generation cationic 
dendrimers, [29],[33] whilst also displaying rapid transport across epithelial barriers. [34]

Various studies have repeatedly demonstrated that the transport of PAMAM dendrimers across epithelial barriers is a function of their concentration, surface charge and their molecular size, especially at higher generations.[29-31, 35] Cationic PAMAM dendrimers terminated with $\mathrm{NH}_{2}$ have consistently demonstrated a detrimental effect on cell barrier integrity in-vitro. This is highlighted by their effect of decreasing the transepithelial electrical resistance (TEER) and increasing in the transport of paracellular markers such as ${ }^{14} \mathrm{C}$-mannitol, especially with cationic dendrimers with higher generations. In contrast, TEER and ${ }^{14} \mathrm{C}$-mannitol transport were not influenced by PAMAM dendrimers terminated with $\mathrm{OH}$ groups and $\mathrm{COOH}$ groups;[36] These studies point to an optimum size and charge of PAMAM dendrimers that can effectively translocate drug molecules across the epithelial barrier of the kidney and gut. Conversely, a systematic correlation between the effect of charge, size, and degree of surface modification of these dendrimers on their permeability across the intestinal barrier have not been assessed.

The objective of this work is to investigate the physicochemical properties of novel PAMAM dendritic carboxylate-terminated molecules with fluorescent cores and their transepithelial transport using an in-vitro model.

\section{Materials and methods}

\subsection{Materials}

Precursors were purchased from Sigma-Aldrich or Acros Organics/Fisher Scientific and were used without further purification. Anhydrous dichloromethane was obtained by distillation over calcium hydride under nitrogen atmosphere. All reactions involving air/moisture sensitive reagents were performed in oven-dried glassware, under a nitrogen atmosphere. TLC analysis refers to analytical thin layer chromatography, using aluminum-backed plates coated with Merck Kieselgel 60 GF254. Flash chromatography was performed on silica gel 60A (3570 micron) chromatography grade (Fisher Scientific). Melting points were recorded using a Gallenkamp Melting Point Apparatus. Infrared spectra were recorded in the range 4000-600 $\mathrm{cm}^{-1}$ using a Perkin-Elmer 1600 series FTIR instrument either as a thin film or as a nujol mull between sodium chloride plates. UV Absorption spectra were recorded on a JASCO (V-570) UV/Vis/NIR spectrophotometer. ${ }^{1} \mathrm{H}$ and ${ }^{13} \mathrm{C}$ NMR spectra were recorded in $\mathrm{MeOD}$ or $\mathrm{CDCl}_{3}$ 
solution (as stated) using an Avance Bruker DPX 400 instrument (400 MHz) or an Avance Bruker DPX $500(500 \mathrm{MHz})$. All chemical shifts $(\delta)$ are reported in ppm with referenced to TMS $(\delta=0.0)$. Low-resolution mass spectrometric data were determined using a Fisons VG Platform II quadrupole instrument using electrospray ionisation (ES) unless otherwise stated. High-resolution mass spectrometric data were obtained in electrospray (ES) mode on a Waters Q-TOF micromass spectrometer. Matrix Assisted Laser Desorption - time of flight Mass Spectrometry (MALDI-TOF) is available using a Waters Maldi Micro MX research grade instrument.

\subsection{Methods}

\subsubsection{Preparation of fluorescent PAMAM-based dendritic Molecules}

The Synthetic procedures and characterizations of the fluorescent PAMAM-based dendritic carboxylic salt molecules G0.5, G1.5 and G2.5 (Figure 1) are presented in supplementary information (SI).

\section{Figure 1}

\subsubsection{Pulsed-Gradient Spin-Echo NMR (PGSE NMR)}

PGSE-NMR measurements were conducted on a Bruker AMX360 NMR spectrometer using a stimulated echo-sequence [37]. Dendron samples were prepared in $\mathrm{D}_{2} \mathrm{O}$ at concentrations ranging from $9.0-23 \mathrm{mM} \mathrm{L}^{-1}$ and all measurements made at $37{ }^{\circ} \mathrm{C}$.

\subsubsection{Self-diffusion coefficient (Ds).}

The self-diffusion coefficient, $D_{s}$ were extracted by fitting the measured PGSE-NMR peak integral to equation 1 .

$$
A(G, \delta)=A_{0} \exp \left[-\gamma^{2} G^{2}\left(\frac{30 \Delta(\delta+\sigma)^{2}-\left(10 \delta^{3}+30 \sigma \delta^{2}+35 \sigma^{2} \delta+14 \sigma^{3}\right.}{30}\right) D_{s}\right]
$$


where $A(G, \delta)$ is the measured NMR peak integral, $\gamma$ is the magnetogyric ratio, $\Delta$ the diffusion time $(140 \mathrm{~ms}), \sigma$ the gradient ramp time $(250 \mu \mathrm{s}), \delta$ the gradient pulse length $(500 \mu \mathrm{s}<\delta<3$ $\mathrm{ms})$ and $\mathrm{G}$ the field gradient strength $\left(0.5<\mathrm{G}<3 \mathrm{Tm}^{-1}\right)$.

\subsubsection{Hydrodynamic radii $\left(R_{h}\right)$}

The hydrodynamic radius $\left(R_{\mathrm{h}}\right)$ of the three fluorescent PAMAM-COONa terminated $(\mathrm{G} 0.5$, G1.5 and G2.5) dendrons as a function of concentration were investigated by diffusion NMR. The self-diffusion coefficients for each of the three PAMAMs as a function of concentration were fitted to a power series (Figure 2) and extrapolated to infinite dilution, from which the hydrodynamic radius was calculated using the Stokes-Einstein equation (2).[38]

$$
R_{h}=\frac{K_{B} T}{6 \pi \eta D_{s}}
$$

where $\left(k_{B}\right)$ is the Boltzmann constant, (T) is the temperature in Kelvin, $(\eta)$ is the viscosity of the solvent (water) and $\left(R_{h}\right)$ is the hydrodynamic radius of the fluorescent Dendron.

\subsubsection{Permeability studies}

MDCK I and MDCK II cells were seeded at 45,000 cells $/ \mathrm{cm}^{2}$ onto polyester 24-well Transwell@ filters of $0.8 \mu \mathrm{m}$ mean pore size, $0.33 \mathrm{~cm}^{2}$ surface area (Corning Incorporated, Corning, NY). Cells were maintained in T-75 flasks using Dulbecco's Modified Eagle's Medium (DMEM) supplemented with 10\% fetal bovine serum, 10,000 units/mL penicillin, $10,000 \mu \mathrm{g} / \mathrm{mL}$ streptomycin at appropriate growth conditions $\left(37^{\circ} \mathrm{C}, 5 \% \mathrm{CO}_{2}\right.$ and relative humidity). Growth medium was changed every 2 days and cells were passaged at 70-90\% confluency using $0.25 \%$ trypsin/ethylenediamine tetraacetic acid (EDTA) solution. Transport medium consisted of DMEM-F12 Invitrogen. and used for transport experiments 4 days postseeding.

The transport of fluorescent core PAMAM dendrons was investigated in the apical-tobasolateral (A-B) direction (Figure 2) at a donor concentration of $1.0 \mathrm{mM}$. Permeability experiments were conducted in a humidified atmosphere of $37{ }^{\circ} \mathrm{C}$. Samples were collected from the receiver (basolateral) chamber at 30, 60, 90 and $120 \mathrm{~min}$. Samples were then analyzed using a Fluostar fluorometer with fluorescent-core PAMAM dendron samples being detected with an excitation wavelength of $460 \mathrm{~nm}$ and an emission wavelength of $540 \mathrm{~nm}$.

\section{Figure 2}


The apparent permeability $\left(\mathrm{P}_{\text {app }}\right)$ coefficients were calculated as follows:

$$
P_{a p p}=\frac{d Q / d T}{A \cdot C_{0}}
$$

where $A$ is the surface area of the membrane filter $\left(\mathrm{cm}^{2}\right), C_{0}$ is the initial concentration in the donor chamber, and $d Q / d T$ is the rate of transport.

The cell monolayer integrity was monitored using an epithelial voltohmmeter (EVOM $\left.{ }^{\mathrm{TM}}\right)$ to measure the TEER (Trans-Epithelial Electrical Resistance).

\section{Results and discussion}

\subsection{Syntheses}

The fluorescent PAMAM-based dendritic carboxylic salt molecules G0.5 (7), G1.5 (8) and G2.5 (9) (Figure 1) synthesis, following a well-established method,[39, 40] was instigated from the precursor 3-(2-aminoethylamino)-7H-benz[de]benzimidazo[2,1-a]isoquinoline-7-one with exclusive Michael addition of methyl acrylate occurring at the aliphatic primary amine, sequential amidations with ethylenediamine and Michael additions with methyl acrylate followed by alkaline hydrolysis of oligoesters, by stirring with ethanolic $\mathrm{NaOH}$ at room temperature using a previous reported procedure.[41] Synthetic procedures and characterization in more details are presented in supplementary information (SI).

\subsection{Physicochemical characterization studies}

\subsubsection{Pulsed-Gradient Spin-Echo NMR (PGSE NMR)}

PAMAM dendrimers have a flexible and loose structure allowing the interior hydrophilic branches to fold outwards in water. $[42,43]$ The potential aggregation behaviour of the three synthesized fluorescent PAMAM-COONa terminated (G0.5, G1.5, G2.5) was investigated via their absolute self-diffusion coefficients and comparison with their parent homologues. Selfdiffusion coefficients where obtained using PGSE-NMR which is well-suited for studying aggregation.[44]. 


\subsubsection{Self-diffusion coefficient $\left(D_{s}\right)$.}

The obtained $D_{s}$ values for G0.5, G1.5 and G2.5 as a function of concentration are presented in Figure (3). In each case, due to their small size, only a modest decrease in the diffusion coefficient is observed as the concentration increases which is in agreement with the behavior observed for the parent homologues.[44]

\section{Figure 3}

\subsubsection{Hydrodynamic radii $\left(R_{h}\right)$}

The hydrodynamic radius $\left(R_{H}\right)$ of the three dendrons at infinite dilution, were found to be $R_{H}$ $(\mathrm{G} 0.5)=11 \AA, R_{H}(\mathrm{G} 1.5)=15 \AA$, and $R_{H}(\mathrm{G} 2.5)=17 \AA$. These results demonstrate that over this relatively high concentration range ("high" compared to those used in the fluorescence studies), the diffusion coefficients of G0.5, G1.5 and G2.5 do not significantly vary either as a function of concentration, or from the values characterizing their parent homologues, demonstrating the non-aggregating properties of these dendritic molecules. This assumption is supported by our previous studies, [41] where we reported that the emission intensity increased almost linearly with concentration for aqueous solutions of G0.5, G1.5 and G2.5 salts indicating that there was no aggregation-induced self-quenching over the concentration range $0.83-5.75 \times 10^{-4} \mathrm{mM} \mathrm{L}^{-1}$.[41, 44-48] As a result from the these statements, we concluded the fluorescence self-quenching of aqueous solutions of the three PAMAM compound salts (G0.5, G1.5 and G2.5) is caused from Photoinduced Electron Transfer (PeT) intramolecular quenching mechanism involving the amine groups of PAMAM different generation, and it is not from intermolecular quenching mechanism concerning the aggregation or self-association. As the PET process (an electron transfer from the primary amine receptor to the excited state of the fluorophore core) would quench fluorescence emission.[49] However, the possibility of PET happening is very low due to the receptor being a primary amine rather than a tertiary amine for which the PET process is more favoured.[50] This trend is due to the effect of the tertiary amines within the PAMAM substituent, which can interact with the fluorophore core via the PET mechanism (Figure 4)[49] and, as the PAMAM generation increases, the number of amines also increases, which enhances the PET process. [41, 51-53]

\section{Figure 4}




\subsection{In-Vitro permeability studies}

Having demonstrated there is no aggregation in these materials, the study then focused on the investigation of the epithelial transport of these dendrons salts (G0.5, G1.5 and G2.5) across cell barriers, using the widely used epithelial in-vitro cell model MDCK (Madin-Darby Canine Kidney). [54-57] Over 2-6 days, confluent MDCK monolayers form a tight junction network visible by confocal microscopy.[58] MDCK I and MDCK II share very similar properties except that in the expression of some tight junctional proteins, namely claudin-3, resulting in drastic differences in their paracellular restrictiveness. For instance, the MDCK I cell line present high restrictiveness, e.g. transepithelial electrical resistance (TEER) of 5000-5250 $\Omega . \mathrm{cm}^{2}$, while the MDCK II cell line is more permeable with a TEER of 75-85 $\Omega . \mathrm{cm}^{2}$. These cell lines were used to study the effect of both monolayer integrity and dendron size upon transport of these auto-fluorescent dendrons to assess the role of paracellular mechanisms in the transport of these dendritic molecules.

\subsubsection{Permeability of fluorescent core PAMAM dendrons across MDCK I, II cell monolayers.}

PAMAM dendrons with fluorescent cores displayed a size-dependent rate of transport across the modestly restrictive MDCK II cell monolayers (TEER $=75-85 \Omega . \mathrm{cm}^{2}$ ) where $P_{a p p}$ of fluorescent PAMAM dendron G0.5 salt was the highest, followed by G1.5 salt which displayed a 7 -fold slower rate of transport (compared to the G0.5) and finally the G2.5 salt. For the G2.5 salt, the transport rate was too slow to be quantified at all time points, due to being below the limit of quantitation (BLQ) (Table 1; Figure 5). For the highly restrictive MDCK I cells (TEER $=5000-5250 \Omega \cdot \mathrm{cm}^{2}$ ), these PAMAM dendrons displayed a similar trend with the smallest dendron displaying the fastest transport rate (albeit 5 fold slower than across MDCK II), while both G1.5 and G2.5 transport rates were too slow to be quantified, again due to samples at all time points being below the limit of quantitation (BLQ) (Table 1; Figure 5). The maximum possible rate of absorption is calculated for those dendrimers that were too slow to be quantified based on their lower limits of quantitation (LLQ).

The direct correlation between monolayer permeability $\left(\mathrm{P}_{a p p}\right)$ and dendritic size as well as the direct correlation between the transport rate of these dendritic molecules and the restrictiveness of the cell monolayers as determined from TEER measurements indicate a significant role of paracellular mechanisms in the transport of these fluorescent dendrons.

Table 1 


\section{Figure 5}

\subsubsection{Effect on monolayer integrity by measuring transepithelial electrical resistance (TEER)}

The effect of modified fluorescent core PAMAM dendrons upon the epithelial integrity of the two MDCK cell types was determined by measuring of the transepithelial electrical resistance across MDCK monolayers. Measurements where made before and after a 120 minute incubation of the fluorescent dendrons at donor concentrations of $1 \mathrm{mM}$. The cell monolayer integrity was monitored using an epithelial voltohmmeter $\left(\mathrm{EVOM}^{\mathrm{TM}}\right)$ to measure the TEER (Trans-Epithelial Electrical Resistance) at $\mathrm{t}=0$ and $120 \mathrm{~min}$.

For both MDCK I and MDCK II cell line, the TEER studies indicate that the presence of any of the three fluorescents (Figures $6 \& 7$ ).

\section{Figure 6}

\section{Figure 7}

\section{Conclusion}

In conclusion, the physicochemical studies indicate that from the magnitudes of the selfdiffusion coefficients (and inter alia hydrodynamic radii), and their insensitivity to concentration, these amphiphilic dendritic molecules are non-aggregating. The water solubility, high fluorescence at near-neutral $\mathrm{pH}$, and absence of aggregation suggest these new dendrons are promising for further utilization in biological studies.

The transport studies suggest that these PAMAM dendritic molecules are biocompatible with an epithelial barrier model and display an ability to permeate across MDCK cell monolayers in a trend that is a function of both the size of the dendrons and restrictiveness of the barrier which indicates that paracellular mechanisms play a predominant role in their transport across epithelia. These studies pave the way for future detailed mechanistic and morphological studies to elucidate the nature of the interaction of fluorescent core PAMAM dendrons with epithelial cells. 


\section{Declaration of Competing Interest}

The authors declare that they have no known competing financial interests or personal relationships that could have appeared to influence the work reported in this paper.

\section{Acknowledgements}

The authors would like to acknowledge the Egyptian Cultural Affairs \& Missions Sector (ECAMS), Ministry of Higher Education and Scientific Research, Egypt and Cardiff University, the UK, for financial support.

\section{Supplementary data}

These data include the synthetic procedures and characterization of compounds 7-9 and the NMR and MALDI-MS spectrum for compound 6. 


\section{References}

1. Tomalia, D.A. and J.M.J. Frechet, Discovery of dendrimers and dendritic polymers: a brief historical perspective. J. Polym. Sci., Part A Polym. Chem., 2002. 40(16): p. 2719-2728.

2. Lee, C.C., et al., Designing dendrimers for biological applications. Nat. Biotechnol., 2005. 23(12): p. 1517-1526.

3. Tekade, R.K., P.V. Kumar, and N.K. Jain, Dendrimers in Oncology: An Expanding Horizon. Chem. Rev. (Washington, DC, U. S.), 2009. 109(1): p. 49-87.

4. Tomalia, D.A., A.M. Naylor, and W.A. Goddard, Starburst Dendrimers: MolecularLevel Control of Size, Shape, Surface Chemistry, Topology, and Flexibility from Atoms to Macroscopic Matter. Angewandte Chemie International Edition in English, 1990. 29(2): p. 138-175.

5. Bouit, P.-A., et al., Dendron-decorated cyanine dyes for optical limiting applications in the range of telecommunication wavelengths. New J. Chem., 2009. 33(5): p. 964968.

6. Caminade, A.-M., A. Hameau, and J.-P. Majoral, Multicharged and/or Water-Soluble Fluorescent Dendrimers: Properties and Uses. Chem.--Eur. J., 2009. 15(37): p. 92709285.

7. Nakaya, K.-I.T., Toshiyuki; Shirataki, Yoshiaki; Shiozaki, Hisayoshi; Funabiki, Kazumasa; Shibata, Katsuyoshi; Matsui, Masaki., 4-(2-Aminoethylamino)-7Hbenz[de]benzimidazo[2,1-a ]isoquinoline-7-one as a highly sensitive fluorescent labeling reagent for carnitine. Bulletin of the Chemical Society of Japan, 2001. 74(1): p. 173-177.

8. Hayek, A., et al., Conjugation of a New Two-Photon Fluorophore to Poly(ethylenimine) for Gene Delivery Imaging. Bioconjugate Chem., 2007. 18(3): p. 844-851.

9. Talanov, V.S., et al., Dendrimer-Based Nanoprobe for Dual Modality Magnetic Resonance and Fluorescence Imaging. Nano Lett., 2006. 6(7): p. 1459-1463.

10. Stewart, W.W., Synthesis of 3,6-disulfonated 4-aminonaphthalimides. J. Am. Chem. Soc., 1981. 103(25): p. 7615-20.

11. Bouche, C.M., et al., Side-chain electroluminescent polymers. Synth. Met., 1996. 81(2-3, 2nd Japan-France Joint Forum (JFJF'2) on Organic Materials and Optoelectronic Devices, 1995): p. 191-195.

12. Morgado, J., et al., 4-AcNI - a new polymer for light-emitting diodes. Synth. Met., 1998. 95(2): p. 113-117.

13. Zhu, W., et al., Luminescent properties of copolymeric dyad compounds containing 1,8-naphthalimide and 1,3,4-oxadiazole. Synth. Met., 1998. 96(2): p. 151-154.

14. Tian, H., et al., Positive and negative fluorescent imaging induced by naphthalimide polymers. J. Mater. Chem., 2002. 12(5): p. 1262-1267.

15. Grabchev, I., J.M. Chovelon, and X. Qian, A copolymer of 4-N,Ndimethylaminoethylene-N-allyl-1,8-naphthalimide with methylmethacrylate as a selective fluorescent chemosensor in homogeneous systems for metal cations. J. Photochem. Photobiol., A, 2003. 158(1): p. 37-43.

16. Grabchev, I., J.-M. Chovelon, and X. Qian, A polyamidoamine dendrimer with peripheral 1,8-naphthalimide groups capable of acting as a PET fluorescent sensor for metal cations. New J. Chem., 2003. 27(2): p. 337-340.

17. Grabchev, I., et al., Novel heterogeneous PET fluorescent sensors selective for transition metal ions or protons: polymers regularly labeled with naphthalimide. New J. Chem., 2002. 26(7): p. 920-925. 
18. Tian, H., et al., Two-path photoinduced electron transfer in naphthalimide-based model compound. J. Chem. Soc., Perkin Trans. 2, 1999(3): p. 545-550.

19. Gunnlaugsson, T., et al., Towards the development of controllable and reversible "onoff" luminescence switching in soft-matter; synthesis and spectroscopic investigation of 1,8-naphthalimide-based PET (photoinduced electron transfer) chemosensors for pH in water-permeable hydrogels. ARKIVOC (Gainesville, FL, U. S.), 2003(7): p. 216-228.

20. Poteau, X., et al., Fluorescence switching in 4-amino-1,8-naphthalimides: "on-off-on" operation controlled by solvent and cations. Dyes Pigm., 2000. 47(1-2): p. 91-105.

21. Jia, L., et al., A novel chromatism switcher with double receptors selectively for Ag+ in neutral aqueous solution: 4,5-diaminoalkeneamino- $N$-alkyl-l,8-naphthalimides. Tetrahedron Lett., 2004. 45(20): p. 3969-3973.

22. Chanh, T.C., et al., Inhibition of retrovirus-induced syncytium formation by photoproducts of a brominated 1,8-naphthalimide compound. Antiviral Res., 1994. 25(2): p. 133-46.

23. Chang, S.C., et al., 4-Alkylamino-3-bromo-N-alkyl-1,8-naphthalimides: new photochemically activatable antiviral compounds. Bioorg. Med. Chem. Lett., 1993. 3(4): p. 555-6.

24. Chang, S.-C., R.E. Utecht, and D.E. Lewis, Synthesis and bromination of 4alkylamino-N-alkyl-1,8-naphthalimides. Dyes Pigm., 1999. 43(2): p. 83-94.

25. Lewis, D.E., et al., Nonazo naphthalimide dyes, their preparation, and their uses. 1993, (Microbiomed Corp., USA). Application: US

US. p. 46 pp.

26. Baughman, R.G., et al., Two related potent antiviral compounds: 3-bromo-N-butyl-4butylamino-1,8-naphthalenedicarboximide (1) and 4-amino-3-bromo-N-butyl-1,8naphthalenedicarboximide (2). Acta Crystallogr., Sect. C Cryst. Struct. Commun., 1995. C51(6): p. 1189-93.

27. Tao, Z.-F. and X. Qian, Naphthalimide hydroperoxides as photonucleases: substituent effects and structural basis. Dyes Pigm., 1999. 43(2): p. 139-145.

28. Esfand, R. and D.A. Tomalia, Poly(amidoamine) (PAMAM) dendrimers: from biomimicry to drug delivery and biomedical applications. Drug Discovery Today, 2001. 6(8): p. 427-436.

29. Wiwattanapatapee, R., et al., Anionic PAMAM dendrimers rapidly cross adult rat intestine in vitro: a potential oral delivery system? Pharm. Res., 2000. 17(8): p. 991998.

30. El-Sayed, M., et al., Transepithelial transport of poly(amidoamine) dendrimers across Caco-2 cell monolayers. J. Controlled Release, 2002. 81(3): p. 355-365.

31. El-Sayed, M., et al., Influence of surface chemistry of poly(amidoamine) dendrimers on caco-2 cell monolayers. J. Bioact. Compat. Polym., 2003. 18(1): p. 7-22.

32. Chiba, F., et al., Dendritic macromolecules as inhibitors to protein-protein binding. Macromol. Symp., 2010. 287(Macro- and Supramolecular Architectures and Materials): p. 37-41.

33. Malik, N., et al., Dendrimers: Relationship between structure and biocompatibility in vitro, and preliminary studies on the biodistribution of 125I-labeled polyamidoamine dendrimers in vivo. J. Controlled Release, 2000. 65(1-2): p. 133-148.

34. Malik, N., et al., Dendrimers:: Relationship between structure and biocompatibility in vitro, and preliminary studies on the biodistribution of 125I-labelled polyamidoamine dendrimers in vivo. J. Controlled Release, 2000. 65(1-2): p. 133-148. 
35. El-Sayed, M., et al., Transepithelial transport of poly(amidoamine) dendrimers across Caco-2 cell monolayers. Journal of Controlled Release, 2002. 81(3): p. 355365.

36. Jevprasesphant, R., et al., The influence of surface modification on the cytotoxicity of PAMAM dendrimers. International Journal of Pharmaceutics, 2003. 252(1-2): p. 263266.

37. Davies, J.A. and P.C. Griffiths, A Phenomenological Approach to Separating the Effects of Obstruction and Binding for the Diffusion of Small Molecules in Polymer Solutions. Macromolecules, 2003. 36(3): p. 950-952.

38. Stokes, G., Cambridge Philos.Trans.1851,9,8-106.Reprinted in Mathematical and Physical Papers, 2nd ed.; Johnson Reprint Corp.: New York, 1996; Vol. 3, p 1.

39. King, A.S., I.K. Martin, and L.J. Twyman, Synthesis and aggregation of amine-cored polyamidoamine dendrons synthesised without invoking a protection/deprotection strategy. Polym. Int., 2006. 55(7): p. 798-807.

40. Brana, M.F., et al., Synthesis and antitumour activity of new dendritic polyamines(imide-DNA-intercalator) conjugates: potent Lck inhibitors. Eur. J. Med. Chem., 2002. 37(7): p. 541-551.

41. El-Betany, A.M.M., et al., The synthesis and study of fluorescent PAMAM-based dendritic molecules. Tetrahedron, 2013. 69(39): p. 8439-8445.

42. Wang, D. and T. Imae, Fluorescence Emission from Dendrimers and Its $p H$ Dependence. J. Am. Chem. Soc., 2004. 126(41): p. 13204-13205.

43. Wang, B.-B., et al., Poly(amidoamine) dendrimers with phenyl shells: fluorescence and aggregation behavior. Polymer, 2004. 45(25): p. 8395-8402.

44. Bányai, I., et al., Self-diffusion of water and poly(amidoamine) dendrimers in dilute aqueous solutions. Soft Matter, 2013. 9(5): p. 1645-1655.

45. Jackson, C.L., et al., Visualization of Dendrimer Molecules by Transmission Electron Microscopy (TEM): Staining Methods and Cryo-TEM of Vitrified Solutions.

Macromolecules, 1998. 31(18): p. 6259-6265.

46. Uppuluri, S., et al., Rheology of Dendrimers. I. Newtonian Flow Behavior of Medium and Highly Concentrated Solutions of Polyamidoamine (PAMAM) Dendrimers in Ethylenediamine (EDA) Solvent. Macromolecules, 1998. 31(14): p. 4498-4510.

47. Fritzinger, B. and U. Scheler, Scaling Behaviour of PAMAM Dendrimers Determined by Diffusion NMR. Macromolecular Chemistry and Physics, 2005. 206(13): p. 12881291.

48. Sagidullin, A., et al., Self-diffusion of low-generation PAMAM dendrimers with hydroxyl surface groups in solutions: a general regularity. Polymer, 2004. 45(1): p. 165-170.

49. Georgiev, N.I., V.B. Bojinov, and P.S. Nikolov, Design and synthesis of a novel $p H$ sensitive core and peripherally 1,8-naphthalimide-labeled PAMAM dendron as light harvesting antenna. Dyes and Pigments, 2009. 81(1): p. 18-26.

50. Wang, D., T. Imae, and M. Miki, Reprint of "Fluorescence emission from PAMAM and PPI dendrimers [J. Colloid Interface Sci. 306 (2007) 222-227]. Journal of Colloid and Interface Science, 2007. 312(1): p. 8-13.

51. Bissell, R.A., et al., Molecular fluorescent signalling with 'fluor-spacer-receptor' systems: approaches to sensing and switching devices via supramolecular photophysics. Chem. Soc. Rev., 1992. 21(3): p. 187-95.

52. Bissell, R.A., et al., Fluorescent PET (photoinduced electron transfer) sensors. Top. Curr. Chem., 1993. 168(Photoinduced Electron Transfer V): p. 223-64.

53. de Silva, A.P., et al., Signaling recognition events with fluorescent sensors and switches. Chem. Rev. (Washington, D. C.), 1997. 97(5): p. 1515-1566. 
54. Chabner Bruce, A. and G. Roberts Thomas, Jr., Timeline: Chemotherapy and the war on cancer. Nat Rev Cancer, 2005. 5(1): p. 65-72.

55. Tajarobi, F., et al., Transport of poly amidoamine dendrimers across Madin-Darby canine kidney cells. International journal of pharmaceutics, 2001. 215: p. 263-7.

56. Volpe, D.A., Variability in Caco-2 and MDCK Cell-Based Intestinal Permeability Assays. Journal of Pharmaceutical Sciences, 2008. 97(2): p. 712-725.

57. Yellepeddi, V.K. and H. Ghandehari, Poly(amido amine) dendrimers in oral delivery. Tissue barriers, 2016. 4(2): p. e1173773-e1173773.

58. Persil, O. and N.V. Hud, Harnessing DNA intercalation. Trends Biotechnol., 2007. 25(10): p. 433-436.

59. Tumiatti, V., et al., Design, Synthesis, and Biological Evaluation of Substituted Naphthalene Imides and Diimides as Anticancer Agent. J. Med. Chem., 2009. 52(23): p. 7873-7877. 


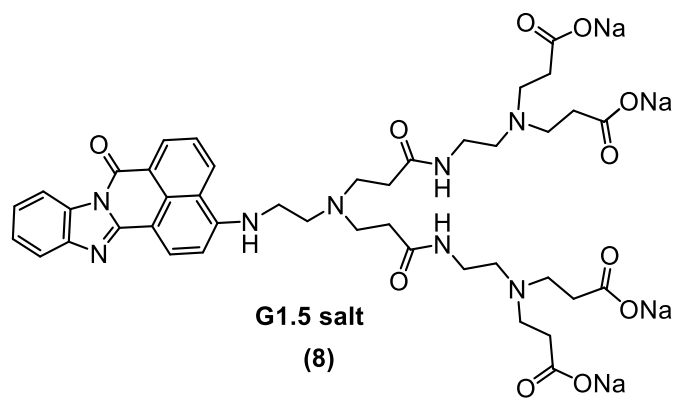

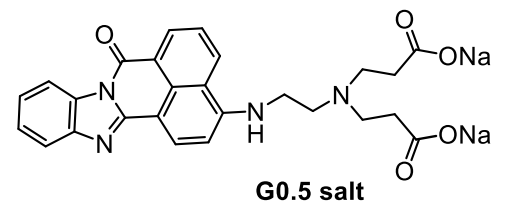

(7)

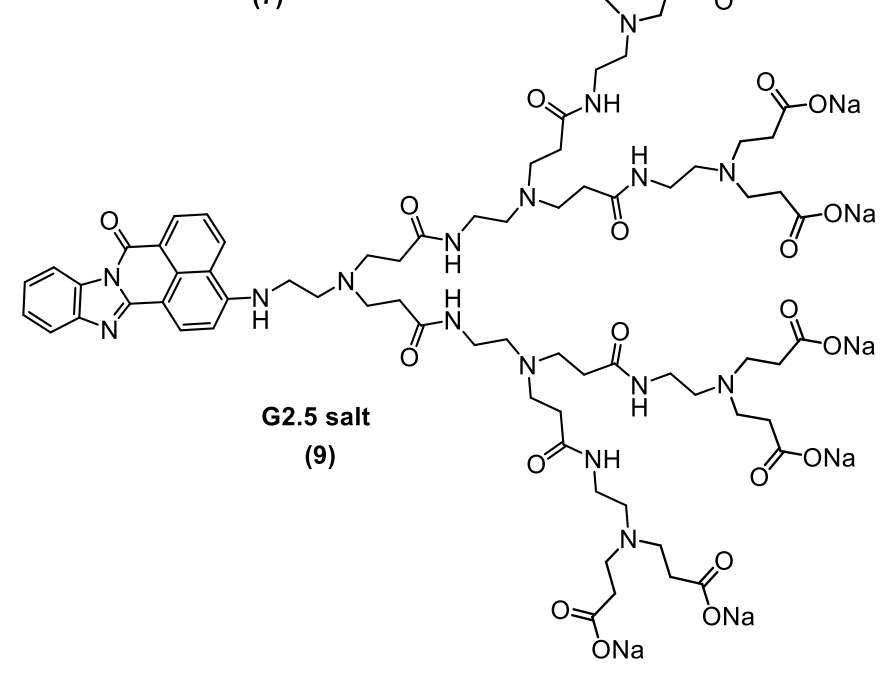

Figure [1]: Chemical structures of compound G0.5, G1.5 and G2.5 salts.

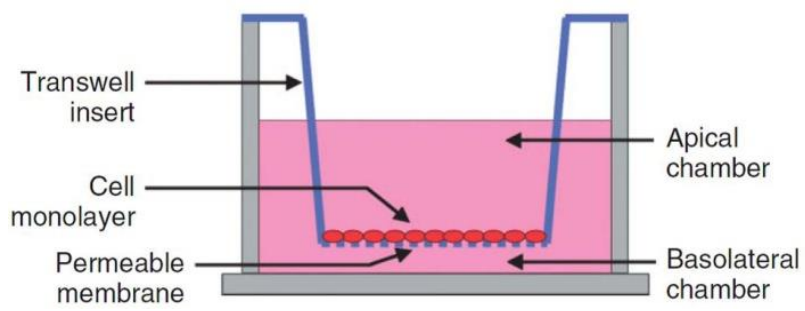

Figure [2]: Schematic of a Transwell® insert showing the separation of apical and basolateral chambers by a permeable membrane with a confluent cell monolayer on the apical surface.[59] 


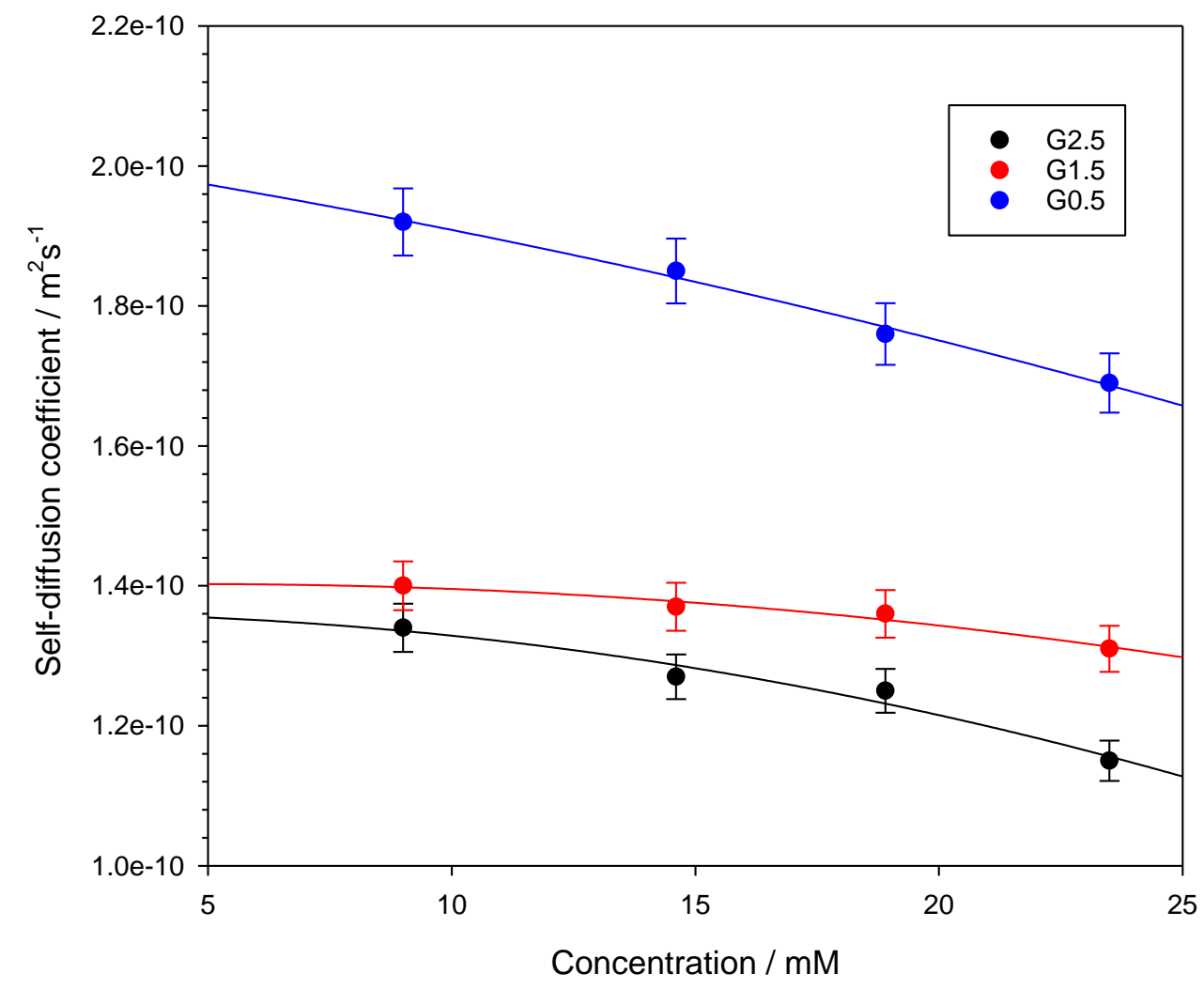

Figure [3]: Self-diffusion coefficient (Ds) of G0.5, G1.5 and G2.5 fluorescent dendron salts in $\mathrm{D}_{2} \mathrm{O}$ at $25^{\circ} \mathrm{C}$ as a function of concentration.

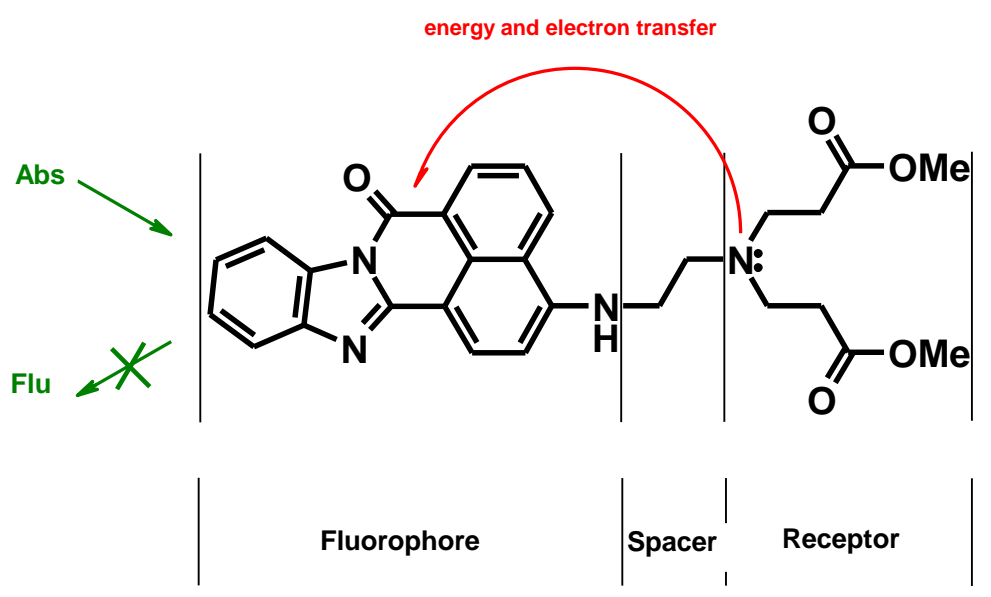

[FC2G0.5]

Figure [4]: Strong fluorescent quenching process resulting from PET in the case of tertiary amine receptor. 


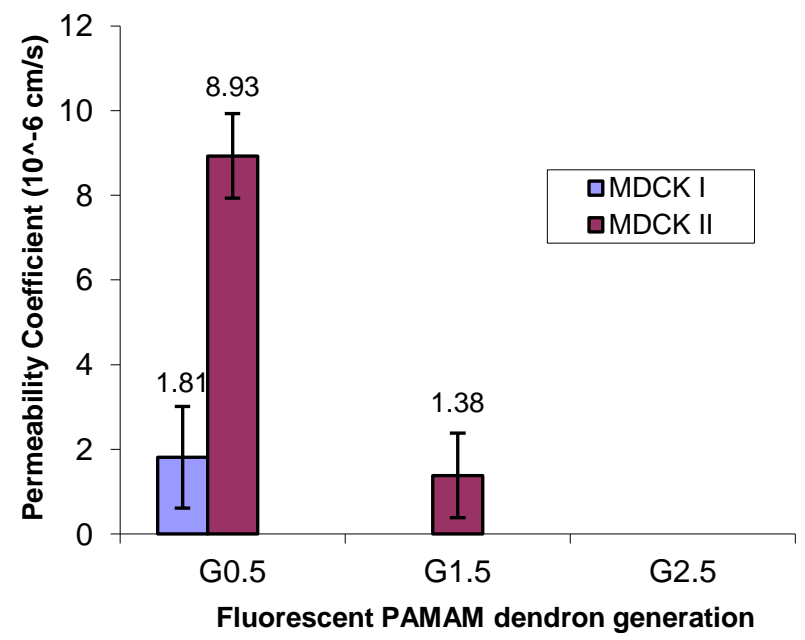

Figure [5]: The permeability of Fluorescent PAMAM dendrons across MDCK I, II cell monolayer in $\mathrm{AB}$ (apical-to-basolateral) directions at concentration $1 \mathrm{mM}$.

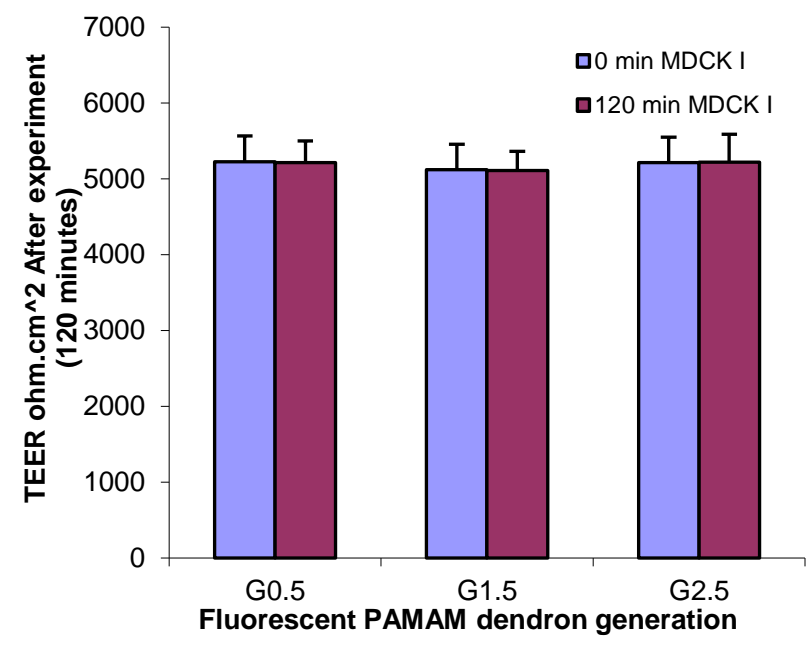

Figure [6]: The effect of 1mM fluorescent PAMAM G0.5, G1.5 or G2.5 on (MDCK I) monolayer integrity as exhibited by their effect on the trans epithelial electrical resistance (TEER) at 0, $120 \mathrm{~min}$. 


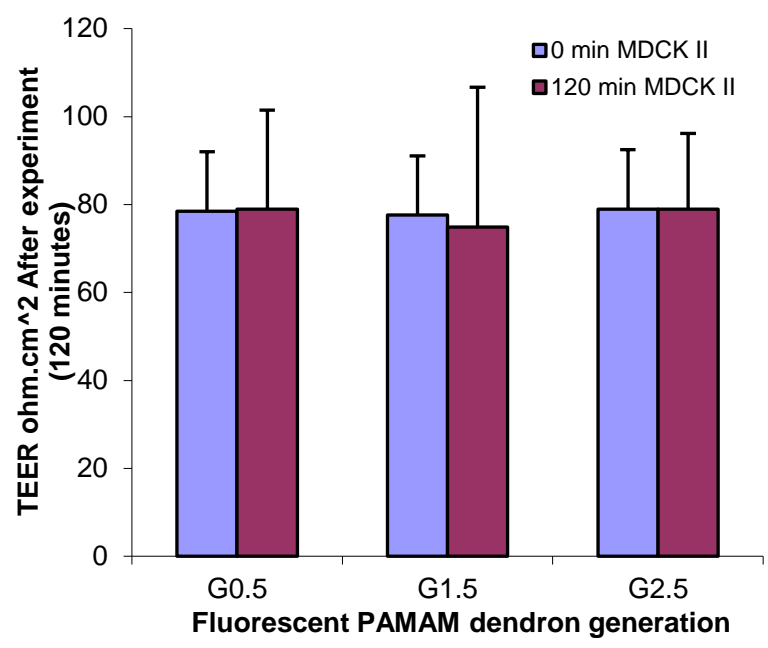

Figure [7]: The effect of 1mM fluorescent PAMAM G0.5, G1.5 or G2.5 on (MDCK II) monolayer integrity as exhibited by their effect on the trans epithelial electrical resistance (TEER) at 0,120 min.

Table [1]:

Permeability Coefficient $\left(10^{-6} \mathrm{~cm} \cdot \mathrm{s}^{-1}\right)$ for dendron generations across

\begin{tabular}{|c|c|c|}
\hline Dendron & $\begin{array}{c}\mathbf{P}_{\text {app }} \text { (MDCK I) } \\
\mathrm{x} 10^{-6} \mathrm{~cm} \cdot \mathrm{s}^{-1} \\
\end{array}$ & $\begin{array}{c}\mathbf{P}_{\text {app }} \text { (MDCK II) } \\
\mathrm{x} 10^{-6} \mathrm{~cm} \cdot \mathrm{s}^{-1} \\
\end{array}$ \\
\hline G0.5 & $1.81 \pm 0.17$ & $8.93 \pm 2.1$ \\
\hline G1.5 & $B L Q<0.58$ & $1.38 \pm 0.24$ \\
\hline G2.5 & $B L Q<0.35$ & $B L Q<3.48$ \\
\hline
\end{tabular}

